Please cite as:

R. Aslam et al., Eur. Phys. J. Special Topics 224, 435-445 (2015)

The final publication is available at link.springer.com

\title{
Weak ac field-induced patterns in vertical deposition of colloids at various evaporation rates
}

\author{
R. Aslam ${ }^{1}$, M. Pichumani ${ }^{2}$, and W. González-Viñas ${ }^{1, a}$ \\ 1 Dept. of Physics and Appl. Math., University of Navarra, Pamplona, Spain \\ 2 Dept. of Nanotechnology, Sri Ramakrishna Engineering College, Coimbatore, India
}

\begin{abstract}
Pattern formation in colloids by weak ac fields in vertical deposition-like configuration at different temperatures has been studied experimentally. At low evaporation (room temperature), the effect of the field leads to the evolution of a one-dimensional array of clusters along the contact line and columnar colloidal dried deposits are obtained at higher evaporation. We investigate the flow dynamics involved in this pattern formation. Homogeneous variation of the contact angle by electrowetting effect becomes unstable and breaks the translational symmetry at the meniscus. Electrokinetic forces together with capillary forces result in the accumulation of particles for pattern formation. The movement of electrically charged colloidal particles is controlled by weak ac electric field even at higher temperatures. We observe the effect of increasing initial particle concentration on the behavior of the clusters for various field frequencies. The average distance between clusters increase monotonically with an increase in the initial particle concentration. We also observe that the average width of columns increases according to the applied field strength.
\end{abstract}

\section{Introduction}

For decades scientists have been studying the effect of external fields on bulk colloidal suspensions to manipulate their interaction, structures and dynamics. The main purpose is to bring these affected colloidal systems into nonequilibrium transitions in a controlled way [1-9]. These transitions lead to macroscopic patterns, and are more complex in nature as their control parameters are influenced by external fields [10, 11]. The knowledge of factors that are restrained by external forces provides basic information about the behavior of colloidal suspensions in these out-of-equilibrium transitions. The application of electric fields is one of the most interesting approaches for manipulating colloidal suspensions in short time scales [12], where different kinds of behavior can be observed, depending on the nature of the field (frequency, field gradients), the particles (polarizability, charge) [13], and the solvent (dielectric constants differences) [14]. Colloidal suspensions with different dielectric constants of solvent and particles subjected to high electric fields show relevant changes in macroscopic properties at the milliseconds scale through electrokinetic effects $[15,16]$. Dynamics

a e-mail: wens@unav.es 
of this kind are too complex to understand as the state of the system becomes unstable and far from the equilibrium. Weak ac field induced-pattern formation in bulk colloidal suspensions using closed cells has already been observed [17]. The vertical deposition technique, is one of the simplest and low cost methods and has been used to form well-ordered colloidal structures [18]. It effectively obtains patterns from very small colloidal particles system where the solvent is evaporating slowly nearing equilibrium. This method is similar to the sedimentation for colloidal particles of larger size [19-21], as the process is long and the rate of sedimentation greater than the rate of evaporation. Therefore, it is not attractive for technological applications. Here, we investigate particle transport and pattern formation in colloidal systems by weak ac fields on diluted suspensions focusing on the interface instead of on the bulk dispersion at different temperatures, by using a vertical deposition-like configuration [22-26].

At room temperature with little volatility solvents, due to instabilities in the contact line by electrowetting action, we get an array of clusters [27]. We verify the involved mechanisms by characterizing the flows of the system using PIV (Particle Image Velocimetry) analysis showing that colloidal particles follow the external field. Local flows are induced in the meniscus through an electrowetting mechanism that leads to the formation of clusters with a well-defined distance between them. This suggests that the long range interaction between particles which causes aggregation is mediated by an electric field through an induced hydrodynamic flow. We investigate the effect of the increasing initial particle concentration on the evolution of clusters subjected to weak ac fields.

For a long time, different techniques have been used to obtain well-ordered dried structures of colloids at the mesoscopic characteristics scales [22,23, 18, 28,5]. Those ordered materials have a large number of technological applications such as sensors, optical coatings, storage devices, optoelectronic devices $[29,30]$. To make vertical deposition technique fruitful for deposition of colloidal particles of large size (micrometer scale), it is better to evaporate the solvent faster by increasing the temperature [31, $32]$. In an attempt to control the process, it has been enhanced by the application of a DC electric field [33].

Here, we report experimental results on weak ac field-induced pattern formation in colloids in vertical deposition-like configuration. We obtain clusters at low evaporation and elongated columnar deposits at higher evaporation. We characterize the behavior of these patterns as a function of applied fields and initial particle concentration.

\section{Experimental set up and procedure}

In our experiments, we use colloidal dispersion of spherical polystyrene particles of diameter $1.3 \mu \mathrm{m}$ (polydispersity: 0.039). These particles are suspended in ultra pure water and their surface charge is $-7 \mu \mathrm{C} / \mathrm{cm}^{2}$. The dispersion $(7.43 \%(\mathrm{w} / \mathrm{w}))$ is provided by Dr. Paulke at Fraunhofer-IAP, Germany. We dilute this dispersion with ultra pure water to low concentrations, from $0.5 \%(\mathrm{w} / \mathrm{w})$ to $1.1 \%(\mathrm{w} / \mathrm{w})$. We use standard glass substrates $\left(1.1 \times 17 \times 18 \mathrm{~mm}^{3}\right)$ with one side $\left(17 \times 18 \mathrm{~mm}^{2}\right)$, coated with a thin conductive layer of Indium Tin Oxide (ITO). The experimental cell is shown in Fig. 1 where the substrates, with conductive side in contact with the suspension, are set vertically in a teflon cell (Fig. 1a). The two substrates are displaced $1 \mathrm{~mm}$ from each other. Ceramic spacers with one of the sides conductive are placed between the substrates for the application of electric field. We use rubber O-ring to avoid leaking of the suspension.

The substrates (as obtained from VisionTek Systems Ltd., United Kingdom) are rinsed with ultra pure water and dried with filtered air blow before mounting in the cell. Weak ac electric fields $(0.8 \mathrm{~V} / \mathrm{mm}$ to $1.2 \mathrm{~V} / \mathrm{mm}$ and $1 \mathrm{~Hz}$ to $3 \mathrm{~Hz})$ of square wave 


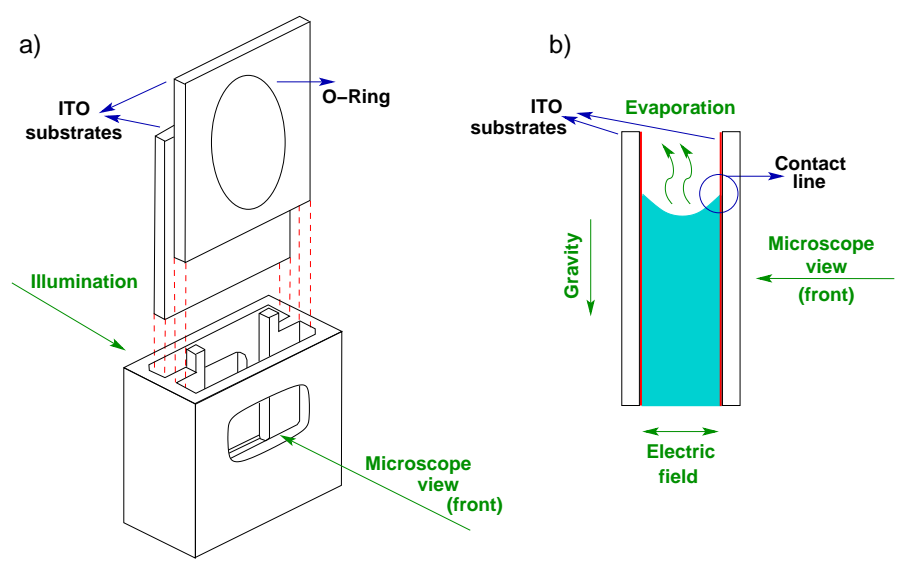

Fig. 1. (Color online) a) Sketch of the unmounted cell, where the illumination and the microscope view is outlined, and b) Sketch of a part of the cell (lateral view). The distance between both substrates is $1 \mathrm{~mm}$.

form are applied perpendicular to the conducting substrates (see Fig. 1b). The experiments are performed at room temperature as well as inside a temperature/humidity chamber at higher temperature. We focus on the region near the contact line using a microscope with long working distance objectives. This microscope is attached to a CMOS camera for capturing frames. A part of the contact line far from lateral boundaries is taken as area of observation and is illuminated by means of a cold light source. The obtained images are further processed by home-made routines of the free software package GNU Octave [34] to measure distances and widths and to extract statistical features.

\section{Experiments}

To illustrate the micrographies that result from both kind of experiments, we show in Fig. 2a a front view sample obtained in the low evaporation experiment. The onedimensional array of clusters (portion of the field of view) can be seen near the contact line, which separates air and colloid on the nearest substrate to the microscope. While we show in Fig. 2b a front view sample obtained in the experiment of high evaporation. A recently deposited (dried or almost dried) vertical column of colloidal particles can be seen in the center of the figure.

As soon as the dispersion $(100 \mu \mathrm{l})$ is poured into the cell at room temperature, the electric field is applied and images are saved sequentially. The particles start to move to the contact line (Fig. 1b, circle) through electrokinetic and capillary forces, where they accumulate. The particles come closer to each other and they group to form clusters and continue to grow till they stabilize their size (Fig. 2a). These clusters start to dissociate [27] after some time (see section 4.2).

Keeping all the experimental conditions unchanged, we also perform experiments in a chamber at fixed temperature and humidity $\left(63^{\circ} \mathrm{C}\right.$, and below $\left.30 \% \mathrm{RH}\right)$ to obtain colloidal dried deposits by applying weak ac fields during the process, at different initial particle concentrations. Fig. 3 shows different stages during the experiment, after the dispersion $(100 \mu \mathrm{l})$ is poured into the cell and the electric field is applied. Particles move toward the meniscus by advection-like flows due to faster evaporation of the solvent and concentrate there. Surface forces trap them into the meniscus. Then these particles come closer to each other, building up the capillary action to begin 

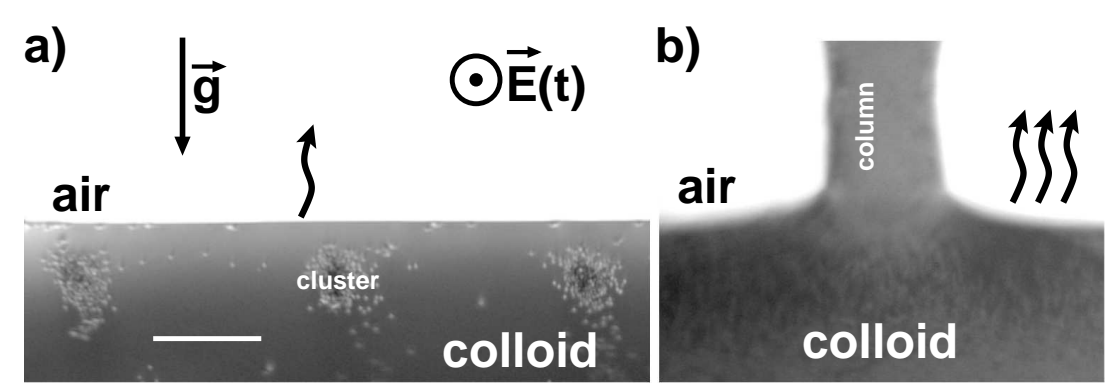

Fig. 2. Schematics of the front view (close to nearer substrate to the microscope, see Fig. 1) for the experiments of (a) clusters with low evaporation (wavy arrow) and (b) columns with high evaporation (wavy arrows). Gravity is downward and contact line separates the colloid and the air. Electric field is applied perpendicular to the substrate and evaporation is perpendicular to the colloid surface in contact with the air. The scale bar is $50 \mu \mathrm{m}$. The conditions are the following: a) Field $1 \mathrm{~V} / \mathrm{mm}$ and $1.4 \mathrm{~Hz}, 0.9 \%$ (w/w) concentration, at room temperature. Clusters near dissociation (see text) b) Field $1 \mathrm{~V} / \mathrm{mm}$ and $1.8 \mathrm{~Hz}, 0.5 \%$ $(\mathrm{w} / \mathrm{w})$ concentration, at $63^{\circ} \mathrm{C}$. Column recently formed (see text).

dried deposits (Fig. 3a) on pinning sites which can be dust particles or substrate small heterogeneities. External fields continue to provide more particles toward the contact
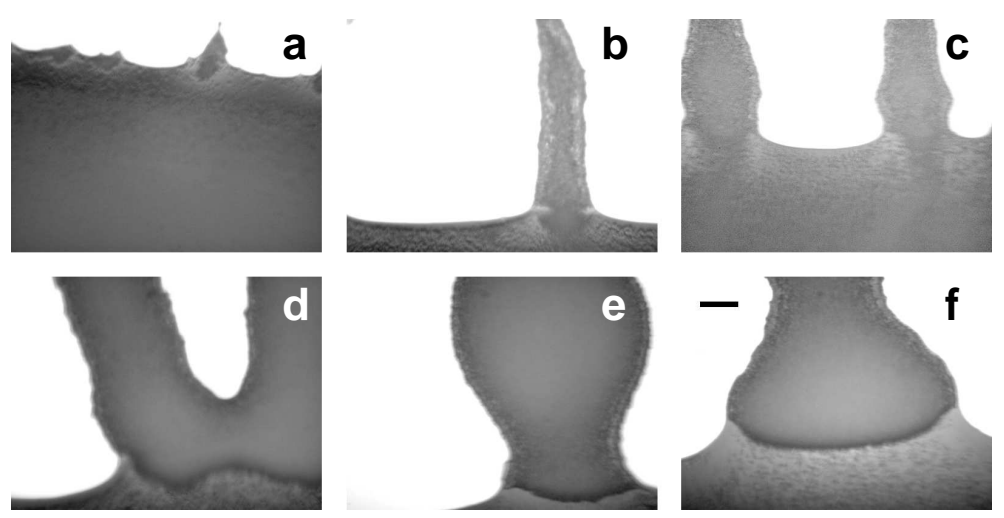

Fig. 3. Front view of the contact line where frames a-f are captured at various increasing times, show different characteristics of the deposition process, at $1 \mathrm{~V} / \mathrm{mm}, 1.8 \mathrm{~Hz}$, concentration $-1.1 \%(\mathrm{w} / \mathrm{w})$ at $63^{\circ} \mathrm{C}$. Scale bar is $50 \mu \mathrm{m}$. Subplots a-f are explained in the text.

line through electrokinetic effect to form elongated columnar deposits (Fig. 3b). When two columns are closer enough to each other to be able to distribute efficiently the incoming particles from the bulk (Fig. 3c), they are not stable and after some time merge together (Fig. 3d). When the columns are stable in size and disposition, they maintain their average width oscillating in correlation with long range flows, that are unrelated to the short period of the applied electric field (Fig. 3e,f). 


\section{Low evaporation rate and evolution of the clusters}

\subsection{PIV analysis}

To demonstrate the response of the system to the applied field, we study the flowdynamics responsible for the aggregation of particles at the contact line with PIV. In Fig. 4, we show the flows near the contact line in the presence of clusters, at $1 \mathrm{~V} / \mathrm{mm}$ and $1.4 \mathrm{~Hz}$ using $0.9 \%$ (w/w) concentration. Subplot (a) shows a bright field

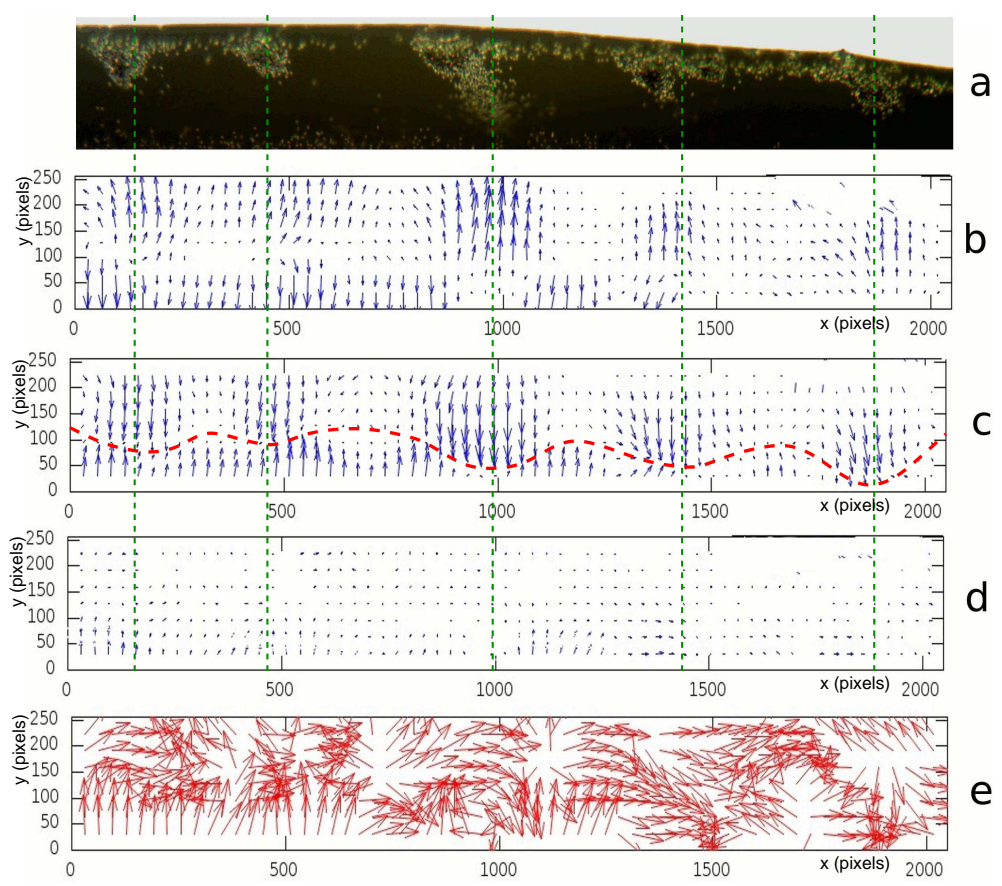

Fig. 4. (Color online) Front view of the flow at the end of the experiment, showing different stages in half cycle of applied field $(1 \mathrm{~V} / \mathrm{mm})$, following field frequency $(1.4 \mathrm{~Hz})$. Dashed wavy line in (c) is a guide to the eye for the stagnation line on the substrate. Vertical dashed lines are guides to the eye which correlate maximum absolute value of vertical velocity (b and c) with the minima of the stagnation line (c) and with the position of the clusters (a). Concentration is $0.9 \%(\mathrm{w} / \mathrm{w})$. Subplots (a-e) are explained in the text. Field of view is $0.67 \mathrm{~mm}=2048$ pixels.

image (field of view is $0.67 \mathrm{~mm}$ ) at a point of the square wave cycle of the applied field, which we set as time-origin of the cycle, (b) is the PIV (cross correlation mean filtered) analysis of (a) at time $t=0,(\mathrm{c})$ is at $t=\frac{T}{2}$, (d) or (e) is the state at $t=\frac{T}{4}$ in between (b) and (c). These different subplots (b-d) indicate that such flows follow the applied field. Meanwhile, during the change in the flow direction, the velocity field is relatively small (Fig. 4d or Fig. 4e, where (e) is the normalized form of (d)).

\subsection{Discussion}

The flow dynamics characterized by PIV analysis (Fig. 4) can be further explained by the microscopic lateral view sketch near the contact line (Fig. 5). The electrokinetic 


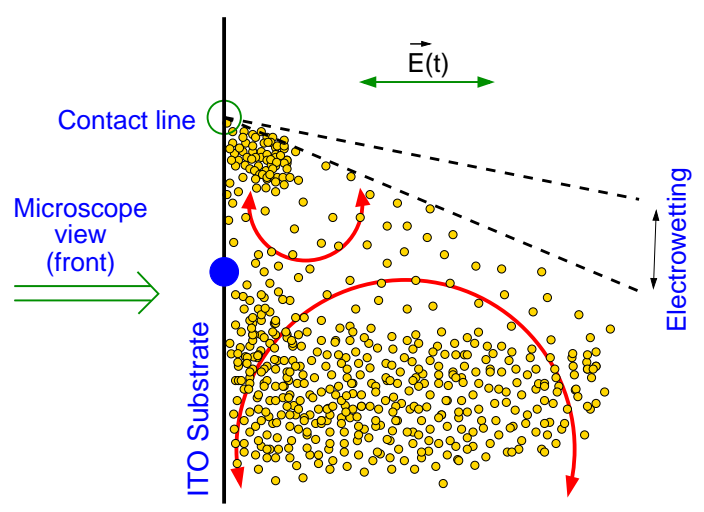

Fig. 5. (Color online) Lateral view sketch of short ranged perpendicular flows following the applied field frequency. States b and c of Fig. 4 correspond to the cases where top curved (red) double arrow points clockwise and counterclockwise, respectively. Top and bottom curved arrows show local flows induced directly by the electric field and oscillate out-ofphase (clockwise versus counterclockwise). The remaining state d of Fig. 4 corresponds to the time when curved arrows change their direction. The up and down variation in the contact angle (electrowetting effect) is shown by the straight double arrow. The open (green) circle represents a point of the contact line. The filled (blue) circle is a point of the stagnation line shown in Fig. 4c.

and surface forces concentrate charged particles in the meniscus. By applying electric field to the system, the variation in the contact angle due to an electrowetting effect generates short range closed flows (perpendicular to the substrate in the meniscus, Fig. 5, curved double arrows), that give rise to instabilities in certain range of the control parameters, like frequency (from $1 \mathrm{~Hz}$ to $3 \mathrm{~Hz}$ ), field strength (from $0.8 \mathrm{~V} / \mathrm{mm}$ to $1.2 \mathrm{~V} / \mathrm{mm}$ ) and initial particle concentration (from $0.5 \%(\mathrm{w} / \mathrm{w})$ to $1.1 \%(\mathrm{w} / \mathrm{w})$ ). This instability breaks the translation symmetry along the contact line and results in an accumulation of particles as a one-dimensional cluster array [27].

Electrophoresis coupled with capillary forces supply more particles to the meniscus [35]. Surface forces trap particles in the meniscus building up the capillary action to attract more particles [36], which increases the size of the basin of attraction. These particles start to accumulate near the contact line in the form of groups. The affected basins of attraction of these groups interact and rearrange according to the availability of particles. This leads to clusters of stable size. These clusters oscillate and follow the applied fields. Through this oscillation of bigger clusters and due to low evaporation, with time the contact line recedes that results in a sudden decrease in the distance between the particle concentrated regions near the contact line and the PPZ (Particle Pool Zone, see [32]). Initially, these two regions are separated by a long distance (Fig. 6a), that gradually decreases (Fig. 6b,c) until the clusters start to dissociate due to interaction with PPZ and the enhancement of convection-like long scale flows related to evaporation.

Emerging groups of particles are spatially distributed and separated initially by a distance $\lambda_{0}$, which corresponds to the wavelength of the instability mentioned above (see $\lambda_{0}$ depicted in Fig. 6a, although it has evolved from previous images where $\lambda_{0}$ cannot be seen easily). The characteristic length $\bar{\lambda}$ defines the average distance between the clusters when they stabilize. An individual $\lambda$ has been represented in Fig. 6b. The influence of the applied field at fixed concentration $0.5 \%(\mathrm{w} / \mathrm{w})$ on the behavior of clusters has been observed in Fig. 5 of [27] indicating the effect of increasing applied field frequencies on characteristic length $(\bar{\lambda})$ for different strengths. 

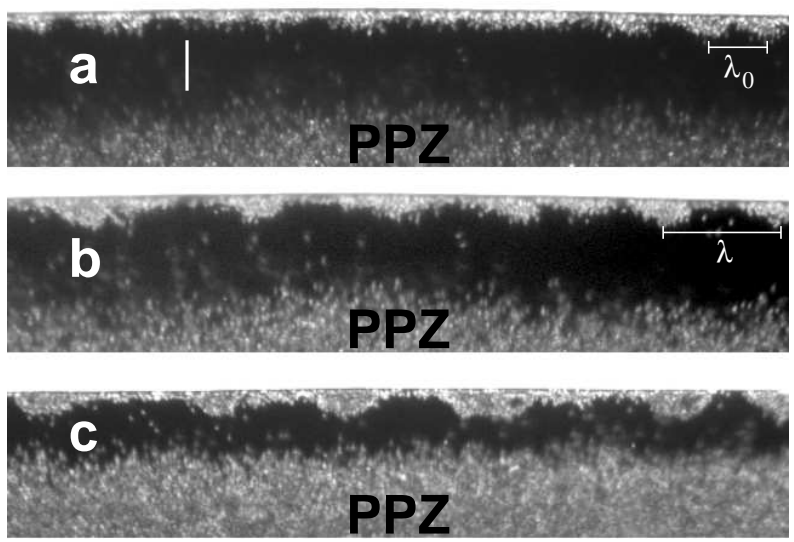

Fig. 6. Front view of low evaporation experiment at different times. Concentration is $0.5 \%$ $\left(\mathrm{w} / \mathrm{w}\right.$ ) and the applied field is $1 \mathrm{~V} / \mathrm{mm}$ and $1.4 \mathrm{~Hz}$. A realization of $\lambda$ and $\lambda_{0}$ are shown. PPZ refers to the Particle Pool Zone (see [32]). The scale bar is $25 \mu \mathrm{m}$. Subplots (a-c) are explained in the text.

The time required for a volume element to complete a cycle in phase (curved double arrows in Fig. 5) during regular flows can be called recurrence time. The length of the fluid element path increases with an increase in field strength and, for having flows in phase, the frequency has to be increased. Higher applied field strength increases the length of these curved double arrows and consequently each cluster has more particles available. The increased capillarity also increases the size of basin of attraction for fetching more particles for cluster formation. Therefore, the characteristic length (distance between stabilized size clusters) increases as the electric field strength does.

\subsection{Effect of Concentration}

We also report the effect of increasing initial particle concentration on the involved mechanism. Fig. 7 shows its influence on the characteristic length $(\bar{\lambda})$ of clusters for different frequencies at fixed field strength of $1 \mathrm{~V} / \mathrm{mm}$. It increases monotonically with an increase in initial particle concentration. If we consider one applied frequency (e.g. $1.4 \mathrm{~Hz}$ in Fig. 7), at lower concentrations (up to $0.7 \%$ (w/w)), this change in the characteristic length is small. However, we observe a bigger change in the characteristic length for higher concentrations, even when the applied field is unchanged. We have also observed (data not shown) that an increase in concentration and electric field strength affect the size of clusters in the same way, because either there are more colloidal particles or the basin of attraction is larger, thus augmenting the effective number of particles available to form the cluster.

\section{Higher evaporation rate and Columnar depositions}

\subsection{Discussion}

At higher temperature the contact line recedes rapidly due to higher evaporation of the solvent. By advection-like flows, particles move toward the meniscus (Fig. 8a) and concentrate there. Surface forces trap particles into the meniscus. Particles come closer to each other and enhance capillary action. The capillary rise within the voids 


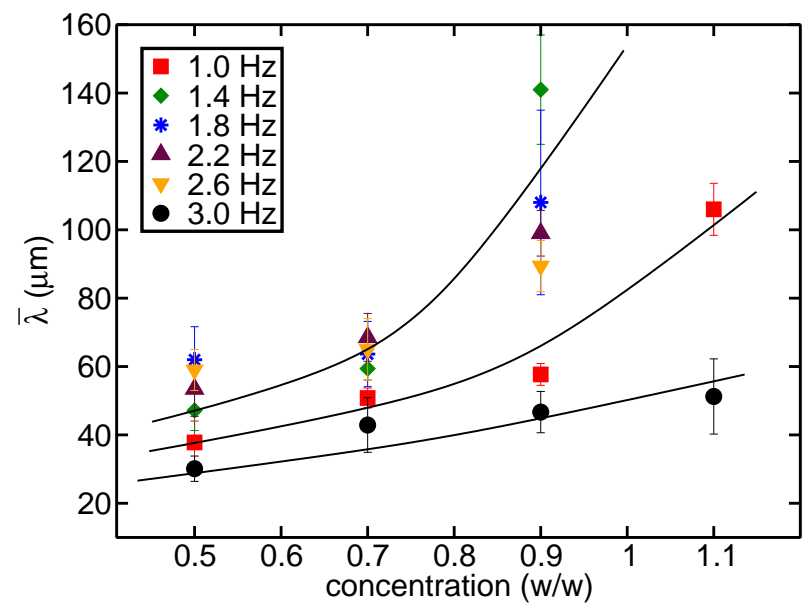

Fig. 7. (Color online) Average characteristic length $(\bar{\lambda})$ as a function of the increasing initial particle concentration for different frequencies at fixed field strength $1 \mathrm{~V} / \mathrm{mm}$. For each frequency, $\bar{\lambda}$ changes monotonically with an increase in concentration. Solid lines are guides to the eye.
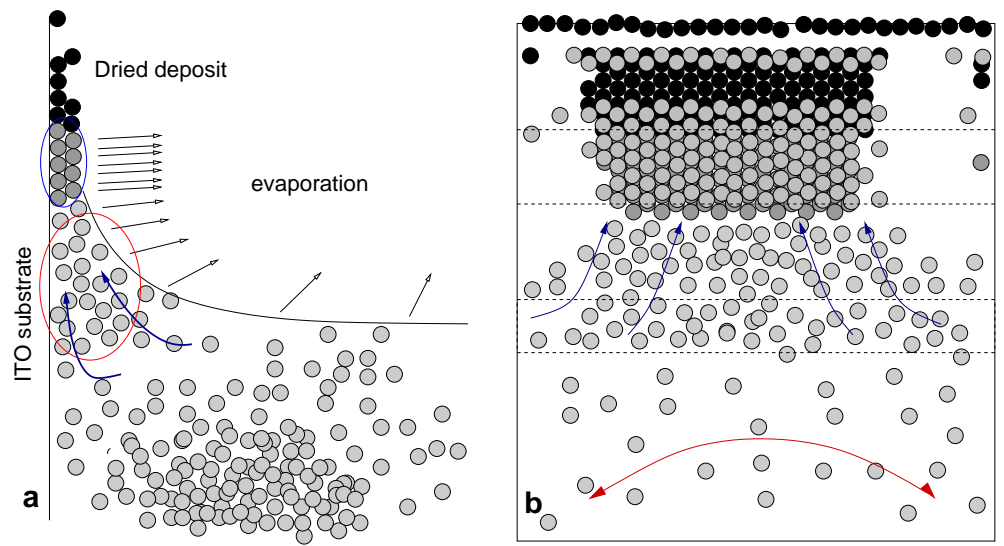

Fig. 8. (Color online) Sketch of the deposition process of a column. Lateral view (a) and front view (b), where capillary flows (upgoing curved arrows), evaporation (straight arrows), and large scale convection flows (double curved arrow) can be seen. For details see the text.

in the initiating columns becomes stronger to provide more particles for the deposition process (Figs. 3a and 8). Once a structure is under construction, the evaporation of the solvent induces new hydrodynamical flows that stimulate further growth of the dried deposits.

The regular order (Fig. 9) which appears in the disposition of the columnar deposits is related to the availability of colloidal particles and, consequently, to the homogeneous character of the colloid at the beginning of the experiment and to the fact that the PPZ is also homogeneous during the experiment. Colloidal particles follow the applied field even at higher temperature (curved double arrows of Fig. 5). Nevertheless, the capillary flows (Fig. 8) overcome them and allow the steady growth of the columns as the contact line recedes.

To identify the role of the external field on colloidal deposits, we carry out the experiment with and without field (Fig. 9). With field, we obtain elongated columnar 


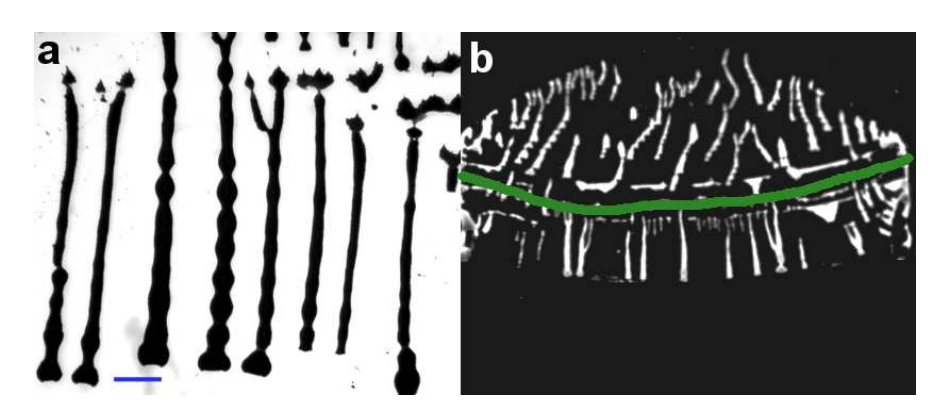

Fig. 9. (Color online) Front view of dried deposits of colloidal particles. (a) Low magnification bright field image. The scale bar is $200 \mu \mathrm{m}$. Concentration $-0.5 \%(\mathrm{w} / \mathrm{w})$. Field during deposition $-1.8 \mathrm{~Hz}$ and $0.8 \mathrm{~V} / \mathrm{mm}$. (b) Image of the deposition (field of view: $12.9 \mathrm{~mm}$ ) with crossed polarizers where deposits above the green line are obtained without field and below it are with applied field. Concentration - $0.7 \%(\mathrm{w} / \mathrm{w})$. Field $-1 \mathrm{~Hz}$ and $1 \mathrm{~V} / \mathrm{mm}$. In both cases, gravity points downward of the images during the deposition.

depositions with regular order (Fig. 9a). Whereas, Fig. 9b shows the dried deposits of the same experiment both without (above the green line) and with (below the green line) field. This indicates that without field we obtain less ordered dried deposits.

\subsection{Effect of field strength}

We investigate the influence of the field strength on the behavior of the dried deposits. Fig. 10 shows that, the characteristic width of columnar depositions is an increasing function of applied field strength at fixed frequency. If there is an increase in applied

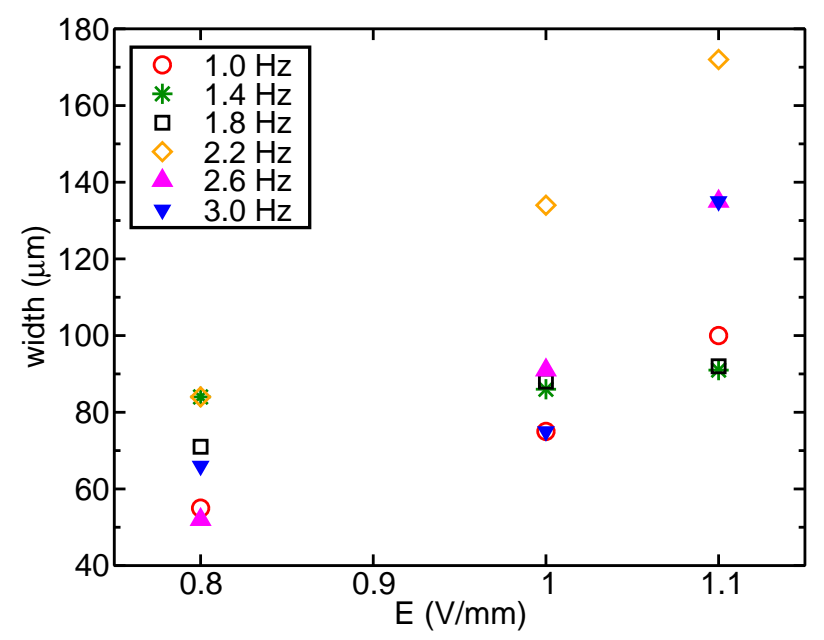

Fig. 10. (Color online) Average width of the columns as a function of the field strength for different frequencies at fixed concentration $-0.5 \%(\mathrm{w} / \mathrm{w})$. For each frequency, width is increasing with an increase in the field strength.

field strength, then the columns cover more region in the affected basin of attraction to fetch more particles for the deposition (Fig. 5). 


\section{Conclusions}

Experimental results on weak ac field-induced pattern formation in diluted colloids have been studied both at room temperature and at higher temperature. Weak ac fields lead to the evolution of clusters at the interface at lower evaporation and elongated columnar deposits are obtained at higher evaporation. We demonstrate the involved mechanisms for this pattern formation in terms of applied fields and initial particle concentration. PIV analysis of experimental data for the evolution of clusters shows that, variation in the contact angle by an electrowetting effect generates short range closed flows giving rise to instabilities in certain range of the control parameters, that break translational symmetry in the meniscus to supply particles there. These particles concentrate in the meniscus and come closer to each other to enhance capillary action. Therefore, electrokinetic forces coupled with capillary forces contribute to the evolution of clusters. A monotonic change in the characteristic length $\bar{\lambda}$ with an increase in the initial particle concentration is observed at various frequencies. Moreover, we observe that, the movement of charged colloidal particles is influenced by weak ac fields even at higher temperature. Such that, external fields keep the connection of the hydrodynamical flows in affected basin of attraction to obtain elongated columnar depositions with regular order. Also, our experimental results report that, the width of dried deposits is associated with applied field strength.

\section{Acknowledgments}

This work is partly supported by the Spanish Government Contract No. FIS201124642. RA and MP acknowledge financial support from the "Asociación de Amigos de la Universidad de Navarra". MP acknowledges the Management, Sri Ramakrishna Engineering College, Coimbatore, India. We thank M. López and J. Burguete for their assistance with PIV analysis, and Olga E. Marlin for carefully reading the manuscript in order to correct grammatical errors.

\section{References}

1. M. Trau, D.A. Saville, I.A. Aksay, Science 272, 706 (1996)

2. T. Gong, D.T. Wu, D.W.M. Marr, Langmuir 19, 5967 (2003)

3. A. Yethiraj, A. Wouterse, B. Groh, A. van Blaaderen, Phys. Rev. Lett. 92, 058301 (2004)

4. A. Yethiraj, Soft Matter 3, 1099 (2007)

5. C. Arcos, K. Kumar, W. González-Viñas, R. Sirera, K.M. Poduska, A. Yethiraj, Phys. Rev. E 77, 050402(R) (2008)

6. Y. Hai-Tao, W. Ming, G. Yi-Xian, Y. Ping, Chinese Phys. B 18, 2389 (2009)

7. M. Giuliani, W. González-Viñas, K.M. Poduska, A. Yethiraj, J. Phys. Chem. Lett. 1, $1481(2010)$

8. A.P. Bartlett, M. Pichumani, M. Giuliani, W. González-Viñas, A. Yethiraj, Langmuir 28, 3067 (2012)

9. M. Pichumani, W. González-Viñas, Soft Matter 9(8), 2506 (2013)

10. M. Cross, P. Hohenberg, Rev. Mod. Phys. 65(3), 851 (1993)

11. D. Wirtz, M. Fermigier, Phys. Rev. Lett. 72(14), 2294 (1994)

12. S. Fraden, A.J. Hurd, R.B. Meyer, Phys. Rev. Lett. 63(21), 2373 (1989)

13. J. Zhou, F. Schmid, Eur. Phys. J. E Soft Matter 36(4), 1 (2013)

14. A.P. Gast, C.F. Zukoski, Adv. Colloid Interface Sci. 30, 153 (1989)

15. A. Yethiraj, A. van Blaaderen, Int. J. Mod. Phys. B 16, 2328 (2002)

16. A. Yethiraj, A. van Blaaderen, Nature 421, 513 (2003)

17. Y. Han, Ph.D. thesis, The University of Chicago (2003) 
18. C. Brinker, Y. Lu, A. Sellinger, H. Fan, Adv. Mater. 11(7), 579 (1999)

19. K. Davis, W. Russel, W. Glantschnig, J. Chem. Soc. Faraday Trans. 87, 411 (1991)

20. H. Míguez, F. Meseguer, C. López, A. Mifsud, J. Moya, L. Vázquez, Langmuir 13, 6009 (1997)

21. P. Segrè, F. Liu, P. Umbanhowar, D. Weitz, Nature 409, 594 (2001)

22. A.S. Dimitrov, K. Nagayama, Langmuir 12, 1303 (1996)

23. P. Jiang, K.S. Hwang, D.M. Mitterman, J.F. Bertone, V.L. Colvin, J. Am. Chem. Soc. 121, 11630 (1999)

24. L.M. Goldenberg, J. Wagner, J. Stumpe, B.R. Paulke, E. Görnitz, Langmuir 118, 3319 (2002)

25. P. Nozar, C. Dionigi, A. Migliori, G. Calestani, L. Cademartiri, Synthetic Met. 139, 667 (2003)

26. D. Norris, E. Arlinghaus, L. Meng, R. Heiny, L. Scriven, Adv. Mater. 16, 1393 (2004)

27. M. Pichumani, M. Giuliani, W. González-Viñas, Phys. Rev. E 83(4), 047301 (2011)

28. F. Meseguer, Colloids and Surfaces A: Physicochem. Eng. Aspects 270-271, 1 (2005)

29. D. Liu, Y. Xiang, X. Wu, Z. Zhang, L. Liu, L. Song, X. Zhao, S. Luo, W. Ma, J. Shen et al., Nano Lett. 6, 2375 (2006)

30. D.J. Norris, Y.A. Vlasov, Adv. Mater. 13, 371 (2001)

31. M. Yoldi, C. Arcos, B.R. Paulke, R. Sirera, W. González-Viñas, E. Görnitz, Mat. Sci. Eng. C-Bio. S. 28, 1038 (2008)

32. M. Giuliani, W. González-Viñas, Phys. Rev. E 79(3), 032401 (2009)

33. M. Giuliani, M. Pichumani, W. González-Viñas, Eur. Phys. J. Special Topics 192, 121 (2011)

34. Octave community, GNU Octave 3.8.1 (2014), www.gnu.org/software/octave/

35. D. Hou, H.C. Chang, Phys. Fluids 18, 071702 (2006)

36. L. Meng, H. Wei, A. Nagel, B.J. Wiley, L.E. Scriven, D.J. Norris, Nano Lett. 6, 2249 (2006) 